\title{
Viabilidade do uso de argilas cauliníticas do Quadrilátero Ferrífero para a indústria cerâmica
}

\author{
(Availability of the kaolin from Quadrilátero \\ Ferrifero for the ceramic industry)
}

\author{
M. G. Peralta-Sánchez, A. M. Morales-Carrera, A. F. D. C. Varajão, M. M. Ferreira \\ Departamento de Geologia, Escola de Minas, Universidade Federal de Ouro Preto, Campus Universitário, \\ Morro do Cruzeiro s/n, Ouro Preto, MG 35400-000 \\ anamc73@gmail.com
}

\begin{abstract}
Resumo
Sete amostras compostas, representativas de três ocorrências de argila caulinítica do Quadrilátero Ferrífero, foram caracterizadas física, mineralógica e quimicamente visando seu uso na indústria cerâmica. Apesar da presença predominante de caulinita, variações nos teores de $\mathrm{Fe}_{2} \mathrm{O}_{3}$ entre 2 a 44\%, refletidos pela presença de goethita e hematita, acarretam diferentes cores que variam entre branco (ACM e DB), amarelo (R5), vermelho amarelado (FS) e vermelho (DV, R1 e AM). Corpos cerâmicos foram queimados a 900 e $1100{ }^{\circ} \mathrm{C}$, sendo que as cores praticamente não mudaram após a queima. Entretanto, somente corpos feitos com DV e R1 cumpriram as especificações técnicas para cerâmica, não apresentando fraturas e atingindo altas resistências à compressão: 96,2 $\mathrm{MPa}$ e 64,2 MPa, respectivamente. Para as outras amostras foram testadas misturas com filito (F) nas proporções de 5, 10, 15, 20 e $25 \%$ e queima a 900 e $1100{ }^{\circ} \mathrm{C}$. Os melhores resultados dos corpos cerâmicos, com ausência de fraturas, resistências à compressão entre 69,15 e $29,6 \mathrm{MPa}$ e que cumpriram as especificações técnicas, foram obtidos nas proporções: $\mathrm{ACM}+15 \mathrm{~F}, \mathrm{DB}+5 \mathrm{~F}, \mathrm{R} 5+5 \mathrm{~F}$, $\mathrm{FS}+5 \mathrm{~F}, \mathrm{AM}+5 \mathrm{~F}$ e queima a $900{ }^{\circ} \mathrm{C}$.
\end{abstract}

Palavras-chave: caulim, filito, cerâmica, Quadrilátero Ferrífero.

Abstract

Seven samples from three kaolin deposits of the Quadrilátero Ferrifero were characterized by physical, mineralogical and chemical analyses in order to determine their use in the ceramic industry. Despite the predominant presence of kaolinite, the variations in the $\mathrm{Fe}_{2} \mathrm{O}_{3}$ content from 2 to $44 \%$, due to presence of goethite and hematite, resulted in different colors, namely: white (ACM and DB), yellow (R5), yellowed red (FS) and red (DV, R1 and AM). Specimens were burned at 900 and $1100^{\circ} \mathrm{C}$ and they almost didn't change color. However, only specimens made with DV and R1 samples reached the technical specifications for the ceramic industry, without fractures and with values of compression strength of 96.2 and $64.2 \mathrm{MPa}$, respectively. The other samples were made by mixing phyllite in the proportions of 5, 10, 15, 20 and 25\% and burned at 900 and $1100{ }^{\circ} \mathrm{C}$. The bricks made with $A C M+15 F, D B+5 F$, $R 5+5 F, F S+5 F, A M+5 F$ and burned at $900^{\circ} \mathrm{C}$ reached the technical specifications for ceramic industry, without fractures and with values of compression strength between 69.15 and $29.6 \mathrm{MPa}$.

Keywords: kaolin, phyllite, ceramic industry, Quadrilátero Ferrífero.

\section{INTRODUÇÃO}

A ampla distribuição de depósitos argilosos caulinítica no Quadrilátero Ferrífero despertou o interesse pela sua pesquisa desde o início do século XIX [1]. Entretanto, apesar dos inúmeros trabalhos [2-10], estes, de caráter genérico, visaram essencialmente a gênese destes corpos ficando o estudo da sua potencialidade para a indústria de cerâmica em um segundo plano. Ressalta-se, portanto a importância dos trabalhos mineralógicos, cristaloquímicos e micromorfológicos de detalhe [11-20] que forneceram uma caracterização detalhada de algumas ocorrências, dados estes imprescindíveis para estudos aplicativos. Neste contexto estudos realizados nos corpos argilosos cauliníticos do depósito de Padre Domingos [18] e, do Morro do
Caxambu [20-23] forneceram uma detalhada caracterização desses corpos com a definição e descrição das diferentes fácies presentes, sendo algumas destas selecionadas para o presente estudo, que objetiva a análise da viabilidade de sua aplicação na indústria cerâmica.

\section{MATERIAIS E MÉTODOS}

Foram estudadas argilas de três localidades no Quadrilátero Ferrífero (Fig. 1): i) depósito de Padre Domingos, localizado no sinclinal Moeda; ii) Morro do Caxambu, no sinclinal Dom Bosco; e iii) taludes ao lado da rodovia BR-040.

No depósito de Padre Domingos as três fácies, previamente definidas [18] como arenito coesivo maciço 


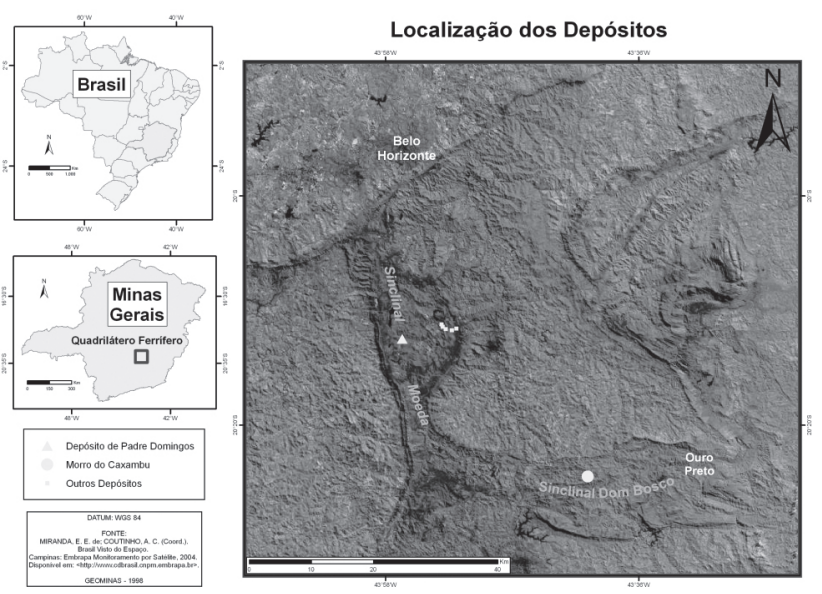

Figura 1: Localização das áreas de estudo no Quadrilátero Ferrífero, Minas Gerais.

[Figure 1: Localization of study areas in Quadrilátero Ferrifero, Minas Gerais, Brazil.]

(ACM), diamictito branco (DB) e diamictito vermelho (DV), foram utilizadas como matérias primas neste estudo. No Morro do Caxambu, das quatro fácies previamente definidas [21-23]: fragmentária, argilosa maciça, nodular e argilosa friável, somente argilosa maciça (AM) e argilosa friável (FS) foram selecionadas devido ao seu maior conteúdo na fração argila $(<2 \mathrm{~mm})$. Amostras de dois taludes (R1 e R5) da rodovia BR-040, também foram coletadas. Adicionalmente, como material aditivo foi utilizado filito $(F)$ da Formação Fecho do Funil, Grupo Piracicaba, Supergrupo Minas, que é o embasamento destes depósitos. Os filitos são utilizados na indústria cerâmica como matéria prima fundente [24] como também na elaboração de massas para revestimentos [25, 26], tanto na forma natural ou beneficiada [27], substituindo parcialmente o feldspato pelo seu alto teor de potássio proveniente da mica muscovita.

A coleta das amostras foi realizada em pelo menos cinco pontos distribuídos da base ao topo de cada fácies/ talude selecionada, com a finalidade de se obter amostras bem representativas e características. Para cada ponto de amostragem, $10 \mathrm{~kg}$ de amostras foram coletadas, secadas a temperatura ambiente, destorroadas e homogeneizadas, sendo, em seguida, preparadas sete amostras compostas, representativas de cada fácies/talude, e designadas com o mesmo nome da fácies/talude de coleta.

Análises granulométricas por peneiramento a úmido, utilizando as peneiras 5, 10, 18, 35, 60, 120, 230 e 400 mesh, que representam os limites dos tamanhos de partículas: grânulo, areia e silte, de acordo com a escala Wenthworth, foram efetuadas. Para essa análise, $50 \mathrm{~g}$ de cada amostra foram misturadas com $500 \mathrm{~mL}$ de uma solução dispersante de metassilicato de sódio $0,1 \% \mathrm{p} / \mathrm{v}$, agitadas e mantidas em repouso por $12 \mathrm{~h}$. Em seguida, as misturas foram novamente agitadas por $5 \mathrm{~h}$ e peneiradas. O material retido em cada peneira foi secado a $70^{\circ} \mathrm{C}$, pesado e calculado o percentual de cada fração. $\mathrm{O}$ tamanho médio das partículas da fração silte fino+argila foi analisado em granulômetro a laser 1064 Cilas.

Difratogramas de raios $\mathrm{X}$, em preparações não orientadas, foram obtidos em um difratômetro Rigaku Geiegrflex D/ Max-B com radiação monocromática $\mathrm{CuK} \alpha$, em faixa angular entre 2 e $70^{\circ}$ e velocidade de $1,2 \% \mathrm{~min}$.

Análises termodiferenciais (ATD) e termogravimétricas (ATG) foram feitas entre 25 e $1100{ }^{\circ} \mathrm{C}$ sob atmosfera de nitrogênio com um equipamento TA SDT 2960, com taxa de aquecimento $20^{\circ} \mathrm{C} / \mathrm{min}$, com alumina como material padrão.

Análises químicas dos elementos maiores e menores foram feitas em equipamento Philips - PANalytical MagiX com amostrador automático PW 2540, equipado com tubo de ródio a $2,4 \mathrm{~kW}$.

$\mathrm{O}$ limite de liquidez e o índice de plasticidade foram determinados de acordo com as NBR 6459 [28] e NBR 7180 [29], respectivamente, utilizando um aparelho Casagrande da Viatest-Pattrol, uma balança Marte AS 2000C e uma estufa FABBE.

Para a preparação dos corpos de prova, as amostras compostas foram peneiradas a \#35 mesh $(0,425 \mathrm{~mm}) \mathrm{e}$ designadas com o mesmo nome da fácies/talude de coleta. Corpos de prova prismáticos $(7,0 \mathrm{~cm} \times 2,0 \mathrm{~cm} \times 1,5 \mathrm{~cm})$ foram confeccionados com $8,5 \%$ de umidade [30], determinada com uma balança Marte ID200, com uma prensa hidráulica Alfred Jamsler. A pressão aplicada variou de acordo com a obtenção de corpos sem fissuras, assim: $8 \mathrm{t}$ para ACM, DB, $\mathrm{R} 5$, FS e AM; 3 t para DV; 2 t para R1 e; 5 t para todas as misturas. A queima, em duas temperaturas, 900 e 1100 ${ }^{\circ} \mathrm{C}$, foi feita em uma mufla Grion com 4 a 5 patamares de aquecimento: $100^{\circ} \mathrm{C} / 12 \mathrm{~h}, 400{ }^{\circ} \mathrm{C} / 2 \mathrm{~h}, 600^{\circ} \mathrm{C} / 2 \mathrm{~h}, 900{ }^{\circ} \mathrm{C} / 3$ h e a $1100^{\circ} \mathrm{C} / 3 \mathrm{~h}$. As temperaturas de queima de 900 e 1100 ${ }^{\circ} \mathrm{C}$ utilizadas nesse trabalho correspondem, respectivamente, ao valor mínimo e máximo de temperatura empregado para a utilização de argilas comuns (argilas cauliníticas) no setor da cerâmica vermelha [31]. Os ensaios de queima preliminares mostraram que somente para as amostras DV e R1 foi possível a obtenção de corpos cerâmicos sem fraturas. Desta forma, para as amostras restantes (ACM, DB, R5, FS e AM) foram feitas misturas com filito $(\mathrm{F})$ nas proporções de 5,10 e $20 \%$. Para ACM ainda foi necessária a adição de $25 \%$ de F, para obter um resultado mais eficiente. Essas misturas foram denominadas de acordo com o nome da amostra e com o teor aditivo, por exemplo, para $\mathrm{ACM}$ : $\mathrm{ACM}+5 \mathrm{~F}, \mathrm{ACM}+10 \mathrm{~F}$, $\mathrm{ACM}+15 \mathrm{~F}, \mathrm{ACM}+25 \mathrm{~F}$. Para cada amostra composta, com aditivo e nos corpos cerâmicos foi determinada a densidade, usando um Multipicnômetro Quantachrome com gás hélio, assim como a superfície específica e a porosidade pelo método de BET (Brunauer, Emmett e Teller) em um analisador automático Quantachrome Nova 1000 com absorção de $\mathrm{N}_{2}$ a $-196^{\circ} \mathrm{C}$ e desgasificação a $60^{\circ} \mathrm{C} / 5 \mathrm{~h}$.

A quantificação da retração linear foi feita relacionando as dimensões dos corpos de prova (em cru) com os corpos cerâmicos (após queima a 900 e a $1100^{\circ} \mathrm{C}$ ). Os valores de absorção de água, de acordo com a norma NBR 8947 [32], foram obtidos relacionando o peso dos corpos cerâmicos secos com o seu peso após fervidos em água durante duas horas. Os ensaios mecânicos de resistência à compressão, de acordo com a norma NBR 6460 [33], foram feitos utilizando a mesma prensa hidráulica da confecção dos corpos de prova. 


\section{RESULTADOS E DISCUSSÃO}

Os resultados obtidos das análises granulométricas (Tabela I) indicam que todas as amostras são predominantemente compostas por silte fino + argila $(\mathrm{SF}+\mathrm{A})$, com mais de $81 \%$, exceto R1 que apresenta 46,5\% dessa fração. O diâmetro médio (DM) das partículas dessa fração varia entre 5,9 a $13,0 \mu \mathrm{m}$; em F é $16,9 \mu \mathrm{m}$.

As amostras da rodovia apresentam os mais altos valores de areia, sendo 18,1\% em R5 e 46,\% em R1. Nas amostras de Padre Domingos essa fração varia entre 5,3 e 7,2\% e nas do Morro do Caxambu entre 4,7 e 9,9\%. No aditivo F essa fração areia atinge valores mínimos (1,6\%). A presença de silte e pequenas proporções de areia nas amostras favorecem a obtenção de algumas características das cerâmicas, tal como não trincamento, retração, vitrificação, etc. [34]. As proporções adequadas para cerâmica vermelha podem variar entre 4 a $7 \%$ de areia, 29 a $50 \%$ de silte e 45 a $62 \%$ de argila [35]. Somente as amostras DV, AM e R1 contêm partículas de tamanho grânulo, atingindo um máximo de $11,8 \%$ em AM. Valores de índice de plasticidade (IP) entre 17,0 e 51,6 (Tabela II), portanto superior a 15 , indicam materiais altamente plásticos [36]. Pode-se notar que o diâmetro médio das partículas finas guarda relação com a plasticidade,

Tabela I - Distribuição de tamanho de partículas das matérias primas e do aditivo.

[Table I - Particle size distribution of the raw materials and of the additive.]

\begin{tabular}{|c|c|c|c|c|c|c|c|c|c|c|}
\hline & \multicolumn{2}{|c|}{ Grânulo } & \multicolumn{5}{|c|}{ Areia } & \multirow{2}{*}{$\begin{array}{c}{ }^{*} \mathrm{SG} \\
\# 400 \\
(\%)\end{array}$} & \multirow{2}{*}{$\begin{array}{c}* \text { SF }+\mathrm{A} \\
<\# 400 \\
(<0,031 \mathrm{~mm}) \\
(\%)\end{array}$} & \multirow[b]{2}{*}{$\begin{array}{r}* * * \mathrm{DM} \\
(\mu \mathrm{m})\end{array}$} \\
\hline Amostra & $\begin{array}{l}\# 5 \\
(\%)\end{array}$ & $\begin{array}{l}\# 10 \\
(\%)\end{array}$ & $\begin{array}{l}\# 18 \\
(\%)\end{array}$ & $\begin{array}{l}\# 35 \\
(\%)\end{array}$ & $\begin{array}{l}\# 60 \\
(\%)\end{array}$ & $\begin{array}{c}\# 120 \\
(\%)\end{array}$ & $\begin{array}{c}\# 230 \\
(\%)\end{array}$ & & & \\
\hline \multicolumn{11}{|c|}{ Padre Domingos } \\
\hline $\mathrm{ACM}$ & 0,00 & 0,00 & 0,14 & 2,13 & 3,06 & 1,34 & 0,51 & 0,21 & 92,61 & 8,8 \\
\hline DB & 0,00 & 0,23 & 0,67 & 1,89 & 2,86 & 1,10 & 0,23 & 0,13 & 92,89 & 13,0 \\
\hline DV & 4,66 & 0,66 & 0,91 & 1,52 & 0,06 & 2,63 & 0,18 & 1,18 & 88,20 & 7,1 \\
\hline \multicolumn{11}{|c|}{ Rodovia } \\
\hline R1 & 0,00 & 0,13 & 1,21 & 8,85 & 13,04 & 12,08 & 11,58 & 6,58 & 46,53 & 5,9 \\
\hline R5 & 0,00 & 0,00 & 0,27 & 6,19 & 7,66 & 3,26 & 0,74 & 0,51 & 81,37 & 12,0 \\
\hline \multicolumn{11}{|c|}{ Morro do Caxambu } \\
\hline FS & 0,00 & 0,00 & 0,07 & 0,19 & 1,23 & 2,86 & 5,53 & 0,06 & 90,06 & 11,9 \\
\hline $\mathrm{AM}$ & 3,97 & 7,81 & 2,02 & 0,68 & 0,45 & 0,18 & 1,40 & 0,15 & 83,34 & 7,9 \\
\hline \multicolumn{11}{|c|}{ Aditivo } \\
\hline $\mathrm{F}$ & 0,00 & 0,00 & 0,00 & 0,00 & 0,00 & 0,01 & 1,57 & 9,36 & 89,06 & 16,9 \\
\hline
\end{tabular}

*SG: silte grosso; **SF+A: silte fino+argila; ***DM: diâmetro médio das partículas inferiores a \# 400

[*SG: coarse silt; **SF+A: small silt + clay; ***DM: medium diameter of the particles above \# 400.]

Tabela II - Características físicas das amostras: limite de liquidez (LL), limite de plasticidade (LP) e índice de plasticidade (IP).

[Table II - Physical characteristics of the samples: liquid limit (LL), plastic limit (PL) and plasticity index (PI).]

\begin{tabular}{lccc}
\hline \multicolumn{4}{c}{ Limites de Atterberg } \\
Amostra & LL & LP & IP \\
\hline ACM & 48,5 & 18,7 & 29,9 \\
DB & 53,8 & 2,2 & 51,6 \\
DV & 51,3 & 33,9 & 17,4 \\
R1 & 28,9 & 7,5 & 21,4 \\
R5 & 49,1 & 15,9 & 33,2 \\
FS & 60,4 & 23,6 & 36,8 \\
AM & 43,7 & 27 & 16,7 \\
\hline
\end{tabular}

pois as amostras com partículas menores refletem em menores índices de plasticidade, enquanto as com partículas maiores, apresentam maiores IP. Já no aditivo não foi possível obter valores de limites de Atterberg nem IP, portanto não apresentou plasticidade.

A mineralogia das sete amostras compostas (Figs. 2 e 3) é similar, predominando caulinita e, secundariamente, quartzo, hematita, feldspato, muscovita e goethita. As análises térmicas diferenciais corroboraram esta composição. $\mathrm{O}$ filito (Figs. 2 e 3) é constituído principalmente por muscovita $(\mathrm{M})$, caulinita $(\mathrm{K})$ e quartzo $(\mathrm{Q})$. Nas misturas podem ser identificados todos os minerais contidos nas argilas e no aditivo F. Nos corpos cerâmicos obtidos a $900^{\circ} \mathrm{C}$ (Figs. 2 e 3) ainda pode ser identificada mica (em menores proporções), quartzo, feldspato e hematita, pois a estrutura cristalina da caulinita foi destruída e, a goethita transformou-se em hematita [37]. Já nos corpos a $1100^{\circ} \mathrm{C}$ (Figs. 2 e 3 ) além do 
(a)

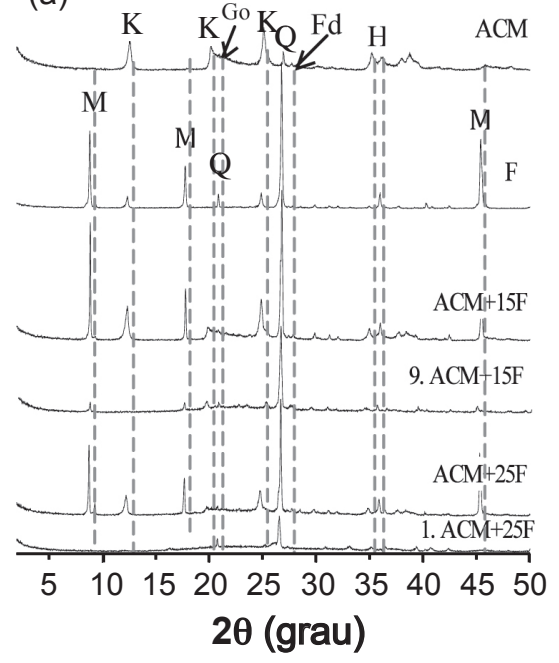

(b)

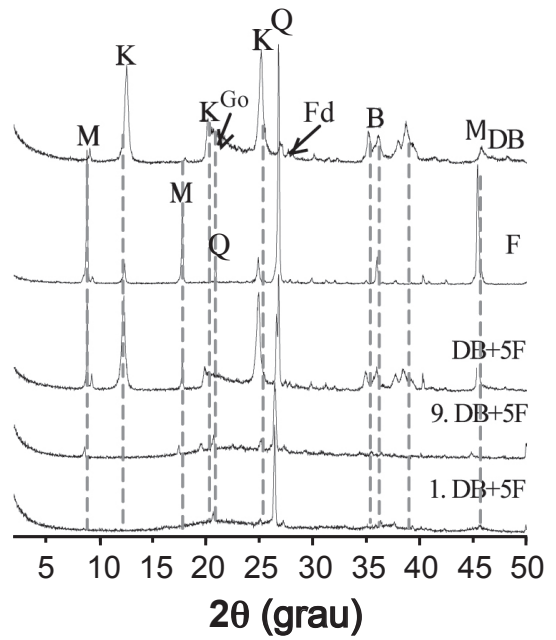

(c)

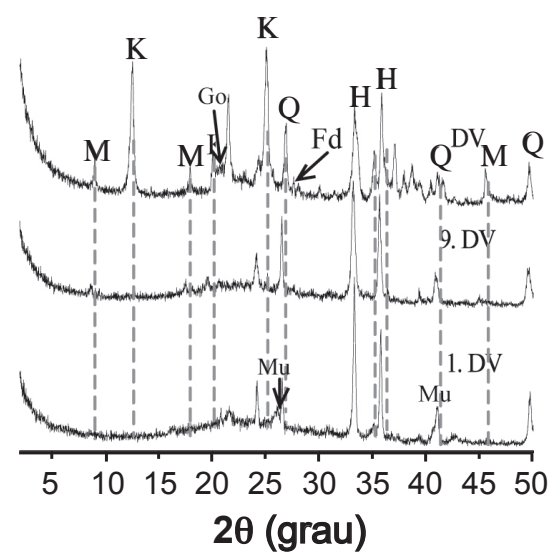

(d)

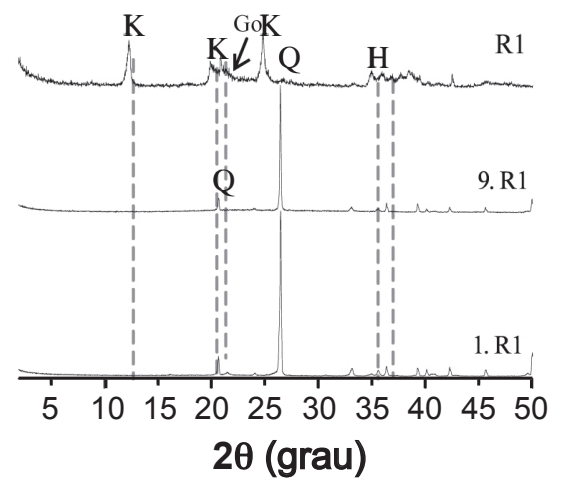

(e)

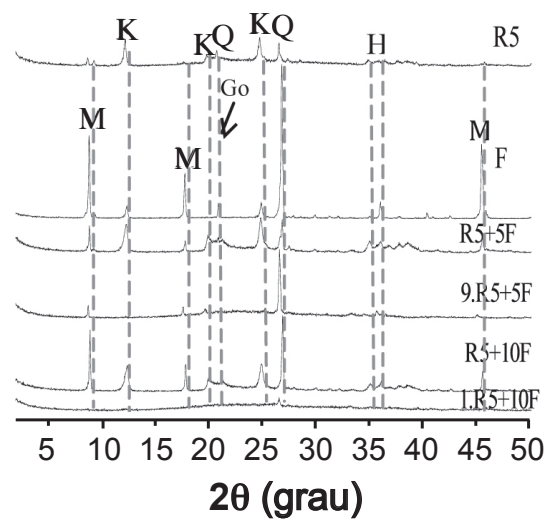

Figura 2: Difratogramas de raios $\mathrm{X}$ das amostras compostas de Padre Domingos (A, B, C), da rodovia (D, E), do aditivo F, das misturas e dos corpos cerâmicos sem fraturas obtidos a $900{ }^{\circ} \mathrm{C}$ (9.) e $1100{ }^{\circ} \mathrm{C}$ (1.). $\mathrm{K}=$ caulinita, $\mathrm{H}=$ hematita, $\mathrm{Q}=\mathrm{quartzo}, \mathrm{Fd}=$ feldspato, $\mathrm{M}=$ muscovita, $\mathrm{Go}=$ goethita, $\mathrm{Mu}=$ mulita .

[Figure 2: X-ray diffraction patterns of the samples from Padre Domingos $(A, B, C)$, road $(D, E)$, of the additive $F$, of the mixtures and of the specimens without fractures burned at $900{ }^{\circ} \mathrm{C}$ (9.) and $1100^{\circ} \mathrm{C}(1$.). $\mathrm{K}=$ kaolinite, $H=$ hematite, $Q=$ quartz, $F d=$ feldspar, $M=$ muscovite, Go $=$ goethite, $M u=$ mullite.]

(a)

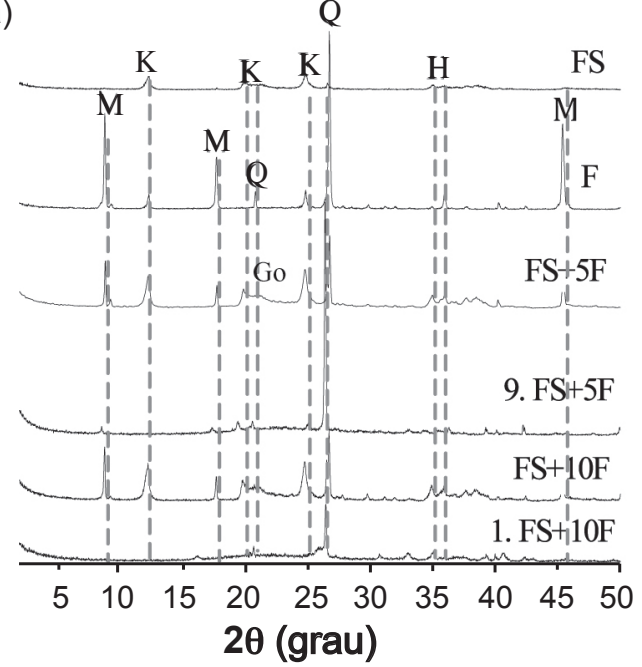

(b)

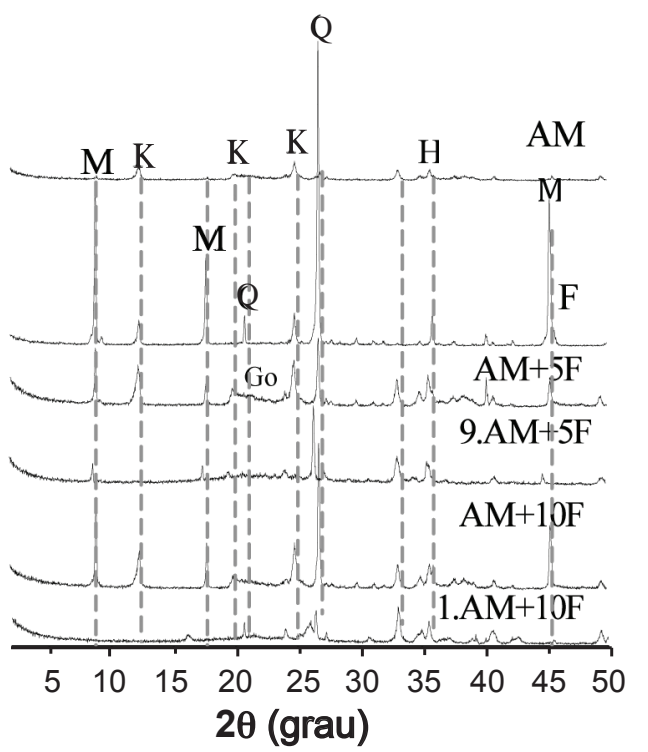

Figura 3: Difratogramas de raios X das amostras compostas do Morro do Caxambu, do aditivo F, das misturas e dos corpos cerâmicos sem fraturas obtidos a $900{ }^{\circ} \mathrm{C}\left(9\right.$.) e $1100{ }^{\circ} \mathrm{C}$ (1.). $\mathrm{K}=$ caulinita, $\mathrm{H}=$ hematita, $\mathrm{Q}=$ quartzo, $\mathrm{Fd}=$ feldspato, $\mathrm{M}=$ muscovita, Go = goethita.

[Figure 3: X-ray diffraction patterns of the samples from Morro do Caxambu, of the additive F, of the mixtures and of the specimens without fractures burned at $900{ }^{\circ} \mathrm{C}$ (9.) and $1100^{\circ} \mathrm{C}$ (1.). $\mathrm{K}=$ kaolinite, $H=$ hematite, $Q=$ quartz, $\mathrm{Fd}=$ feldspar, $M=$ muscovite, Go $=$ goethite.] 
Tabela III - Composição química das amostras e do aditivo.

[Table III - Chemical composition of the samples and of the additive.]

\begin{tabular}{lccccccccccc}
\hline Amostra & $\begin{array}{c}\mathrm{SiO}_{2} \\
(\%)\end{array}$ & $\begin{array}{c}\mathrm{Al}_{2} \mathrm{O}_{3} \\
(\%)\end{array}$ & $\begin{array}{c}\mathrm{Fe}_{2} \mathrm{O}_{3} \\
(\%)\end{array}$ & $\begin{array}{c}\mathrm{K}_{2} \mathrm{O} \\
(\%)\end{array}$ & $\begin{array}{c}\mathrm{TiO}_{2} \\
(\%)\end{array}$ & $\begin{array}{c}\mathrm{MgO} \\
(\%)\end{array}$ & $\begin{array}{c}\mathrm{P}_{2} \mathrm{O}_{5} \\
(\%)\end{array}$ & $\begin{array}{c}\mathrm{MnO} \\
(\%)\end{array}$ & $\begin{array}{c}\mathrm{CaO} \\
(\%)\end{array}$ & $\begin{array}{c}\mathrm{Na}_{2} \mathrm{O} \\
(\%)\end{array}$ & $\begin{array}{c}\mathrm{PF} \\
(\%)\end{array}$ \\
\hline ACM & 45,71 & 34,63 & 2,04 & 1,87 & 1,97 & 0,52 & 0,133 & 0,006 & 0,05 & $<0,001$ & 12,56 \\
DB & 46,38 & 35,20 & 1,58 & 1,34 & 1,72 & 0,33 & 0,065 & 0,003 & 0,03 & 0,014 & 12,53 \\
DV & 24,32 & 18,18 & 44,25 & 1,58 & 0,82 & $<0,01$ & 0,335 & 0,007 & 0,05 & 0,084 & 8,04 \\
$\mathrm{R} 1$ & 70,67 & 12,89 & 10,48 & 0,50 & 0,83 & $<0,01$ & 0,019 & 0,010 & 0,04 & $<0,001$ & 4,58 \\
R5 & 43,14 & 34,69 & 5,37 & 0,54 & 2,02 & 0,04 & 0,083 & 0,017 & 0,05 & 0,013 & 13,27 \\
FS & 40,22 & 27,16 & 17,76 & 0,96 & 1,80 & 0,13 & 0,063 & 0,011 & 0,03 & 0,043 & 10,90 \\
AM & 37,50 & 29,70 & 17,08 & 0,86 & 2,22 & 0,26 & 0,088 & 0,116 & 0,04 & $<0,001$ & 11,46 \\
F & 50,33 & 26,29 & 8,91 & 7,92 & 1,02 & 0,63 & 0,050 & 0,004 & 0,05 & 0,334 & 4,11 \\
\hline
\end{tabular}

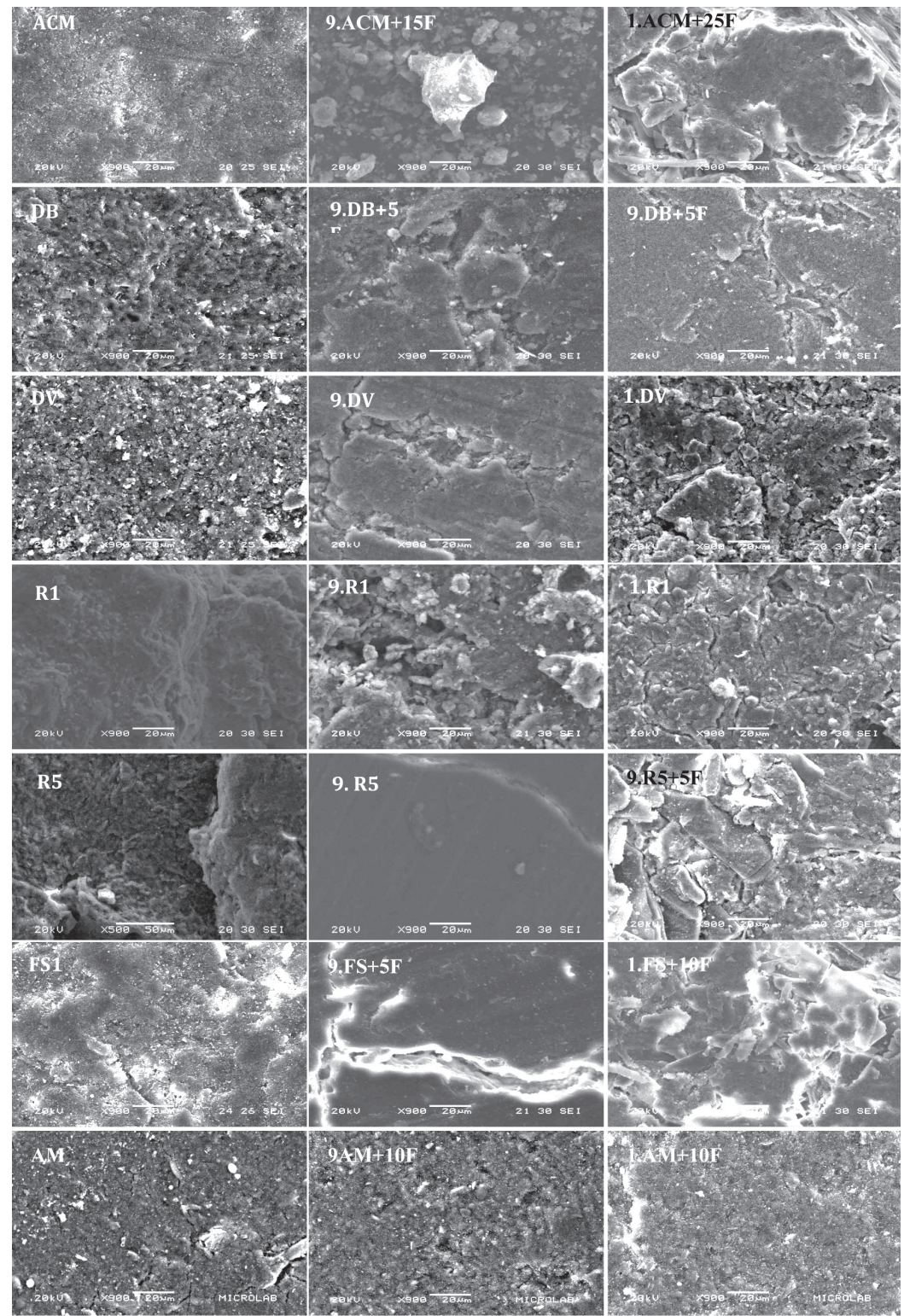

Figura 4: Fotomicrografias de microscopia eletrônica de varredura dos materiais naturais e queimas a 900 e $1100{ }^{\circ} \mathrm{C}$. Com o aumento da sinterização a $1100{ }^{\circ} \mathrm{C}$ o tamanho dos poros aumenta.

[Figure 4: SEM micrographs of the raw materials and specimens burned at 900 and $1100^{\circ} \mathrm{C}$. We can observe the appearance of macropores in the specimens burned at $1100{ }^{\circ} \mathrm{C}$. ] 
quartzo e hematita, aparece a mulita $(\mathrm{Mu})$, sendo esta bem evidente na amostra DV.

A composição química das amostras compostas indica a relação $\mathrm{SiO}_{2} / \mathrm{Al}_{2} \mathrm{O}_{3}$ próxima de 1 (Tabela III). Exceção ocorre em R1 cuja relação é $>5$ devido ao alto conteúdo de quartzo (Fig. 2D). Já no aditivo F, corroborando os dados

Tabela IV - Características físicas das amostras: densidade, superfície específica, porosidade. Somente são apresentadas as misturas queimadas a $900{ }^{\circ} \mathrm{C}(9$.$) e a 1100{ }^{\circ} \mathrm{C}$ (1.) que obtiveram corpos cerâmicos sem fraturas.

[Table IV - Physical characteristics of the samples: density, surface area, porosity. This table only contains the specimens that did not fracture when burned at $900{ }^{\circ} \mathrm{C}$ (9.) and $1100{ }^{\circ} \mathrm{C}$ (1.).]

\begin{tabular}{lccc}
\hline \multicolumn{1}{c}{ Amostra } & $\begin{array}{c}\text { Densidade } \\
\left(\mathrm{g} / \mathrm{cm}^{3}\right)\end{array}$ & $\begin{array}{c}\text { Superfície } \\
\text { especíica } \\
\left(\mathrm{m}^{2} / \mathrm{g}\right)\end{array}$ & $\begin{array}{c}\text { Volume dos } \\
\text { Microporos } \\
\left(\mathrm{cm}^{3} / \mathrm{g}\right)\end{array}$ \\
\hline ACM & 2,70 & 27,63 & 0,0129 \\
9.ACM & 2,86 & 18,50 & 0,0086 \\
9.ACM+15F & 2,99 & 19,57 & 0,0092 \\
1.ACM & 2,85 & 9,92 & 0,0046 \\
1.ACM+25F & 2,95 & 3,54 & 0,0016 \\
DB & 2,66 & 19,54 & 0,0078 \\
9.DB & 2,90 & 15,70 & 0,0073 \\
9.DB+5F & 2,95 & 11,57 & 0,0054 \\
1.DB & 2,84 & 13,68 & 0,0063 \\
1.DB+5F & 3,01 & 1,88 & 0,0009 \\
DV & 3,21 & 36,67 & 0,0164 \\
9.DV & 3,49 & 14,07 & 0,0065 \\
1.DV & 3,62 & 2,64 & 0,0012 \\
R1 & 2,83 & 10,16 & 0,0046 \\
9.R1 & 2,95 & 6,53 & 0,0030 \\
1.R1 & 2,98 & 0,78 & 0,0004 \\
R5 & 2,74 & 26,87 & 0,0124 \\
9.R5 & 2,94 & 20,71 & 0,0096 \\
9.R5+5F & 2,89 & 5,52 & 0,0031 \\
1.R5 & 2,95 & 2,75 & 0,0010 \\
1.R5+10F & 3,09 & 3,44 & 0,0018 \\
FS & 2,69 & 27,04 & 0,0024 \\
9.FS & 2,95 & 11,60 & 0,0055 \\
9.FS+5F & 2,85 & 7,06 & 0,0033 \\
1.FS & 2,99 & 4,31 & 0,0019 \\
1.FS+10F & 2,85 & 3,71 & 0,0015 \\
AM & 2,92 & 22,80 & 0,1067 \\
9.AM & 3,21 & 16,57 & 0,0078 \\
9.AM+5F & 3,18 & 4,66 & 0,0031 \\
1.AM & 3,14 & 1,05 & 0,0005 \\
1.AM+10F & 3,29 & 0,84 & 0,0004 \\
\hline & & & \\
\hline
\end{tabular}

mineralógicos, a sílica é quase o dobro do que a alumina, devido a presença predominante de muscovita. A presença deste mineral reflete no elevado teor em $\mathrm{K}_{2} \mathrm{O}(7,9 \%)$, que influencia diretamente no ponto de fusão das cerâmicas e na composição química das misturas [38, 39]. Foi observado o início da fase liquida a partir dos $900{ }^{\circ} \mathrm{C}$ para misturas de fases tipo caulim-mica, atingindo o intervalo de $1050{ }^{\circ} \mathrm{C}$ a $1100{ }^{\circ} \mathrm{C}$ para misturas tipo mica-caulim [39]. A temperatura de desidroxilação da muscovita pode variar em função dos diferentes polimorfos estruturais ocorrendo de $750{ }^{\circ} \mathrm{C}$ a $1050{ }^{\circ} \mathrm{C}$ [40]. Adicionalmente, este autor mostra que o efeito de moagem pode também interferir na temperatura de desidroxilação deste mineral. No presente trabalho observa-se que a $900{ }^{\circ} \mathrm{C}$ ocorreu fusão parcial da mistura estando ainda presente menores proporções da muscovita, como demonstrado pela diminuição da intensidade do pico a $2 \theta=8,9^{\circ}$; já a $1000^{\circ} \mathrm{C}$ a fusão deste mineral foi total (Figs. 2 e 3 ).

As cores apresentadas pelas amostras [41]: brancas para ACM (5YR-8/1) e DB (5YR-8/1), vermelho amarelado (5YR-5/6) para FS, amarelo (10YR-8/6) para R5 e vermelhas para AM (2.5YR-4/6), R1 (2.5R-4/8) e DV (2.5R-5/8) estão diretamente relacionadas ao conteúdo em ferro. Assim, as amostras de cor branca possuem menores proporções

Tabela V - Valores de absorção de água, retração linear e resistência à compressão das amostras naturais e das misturas com filito para corpos queimados a 900 e $1100{ }^{\circ} \mathrm{C}$. Somente são apresentadas as misturas queimadas a $900{ }^{\circ} \mathrm{C}(9$.) e a $1100{ }^{\circ} \mathrm{C}$ (1.) que obtiveram corpos cerâmicos sem fraturas.

[Table V-Values of water absorption, linear shrinkage and compression strength of natural samples and the mixtures with phylite for burned bricks at 900 and $1100{ }^{\circ} \mathrm{C}$. This table only contains the specimens that did not fracture when burned at $900{ }^{\circ} \mathrm{C}$ (9.) and $1100{ }^{\circ} \mathrm{C}$ (1.).]

\begin{tabular}{lccc}
\hline Amostra & $\begin{array}{c}\text { Absorção } \\
\text { de água } \\
(\%)\end{array}$ & $\begin{array}{c}\text { Retração } \\
\text { linear } \\
(\%)\end{array}$ & $\begin{array}{c}\text { Resistência à } \\
\text { compressão } \\
(\mathrm{MPa})\end{array}$ \\
\hline 9.ACM+15F & 24,88 & 0,28 & 46,20 \\
1.ACM+25F & 16,27 & 3,94 & 31,68 \\
9.DB+5F & 24,05 & 0,98 & 54,64 \\
1.DB+5F & 18,85 & 4,35 & 26,85 \\
9.DV & 25,57 & 0,98 & 26,32 \\
1.DV & 16,79 & 5,48 & 67,22 \\
9.R1 & 13,23 & $-0,57$ & 44,06 \\
1.R1 & 13,44 & $-0,43$ & 46,81 \\
9.R5+5F & 30,40 & 1,55 & 36,47 \\
1.R5+10F & 21,58 & 5,49 & 29,70 \\
9.FS+5F & 29,16 & 1,27 & 39,79 \\
1.FS+10F & 19,13 & 5,34 & 16,50 \\
9.AM+5F & 25,24 & 1,41 & 35,03 \\
1.AM+10F & 14,26 & 7,53 & 16,88 \\
\hline
\end{tabular}


de $\mathrm{Fe}_{2} \mathrm{O}_{3}$ com valores próximos a 2\%, enquanto nas mais vermelhas como DV o conteúdo de ferro é maior a $44 \%$. Após a queima, em geral, não houve mudança de cores, somente DV, R5 e AM apresentaram algumas variações, porém pouco significativas.

A Tabela IV mostra os valores de densidade, superfície específica por BET e porosidade para as amostras compostas, antes e após a queima, e das misturas após a queima. Podese verificar o aumento da densidade após a queima devido às mudanças que ocorrem com a estrutura cristalina dos minerais: a caulinita é destruída e a goethita se transforma em hematita. Este incremento é marcante nas amostras mais ricas em ferro, como DV e AM, que atingem após a queima valores de densidade superiores a $3 \mathrm{~g} / \mathrm{cm}^{3}$ devido a presença predominante de hematita $\left(\mathrm{d}=5,26 \mathrm{~g} / \mathrm{cm}^{3}\right)$ e mulita $\left(\mathrm{d}=3,23 \mathrm{~g} / \mathrm{cm}^{3}\right)$.

Em relação à superfície específica, tanto nos corpos cerâmicos obtidos a partir das amostras compostas, quando com as misturas, observa-se que esses valores diminuem à medida que a temperatura de queima aumenta e, portanto, também diminui a microporosidade. Essas mudanças são mais evidentes nas amostras com maior teor de $\mathrm{Fe}_{2} \mathrm{O}_{3}$ como DV, onde o incremento de temperatura propicia a brusca diminuição da superfície específica acompanhada pela diminuição da microporosidade e aparecimento de macroporos [42] relacionados com o aumento do tamanho de grão da hematita [37, 43-45].

O aumento do tamanho do grão nas amostras, que é uma das características da sinterização [46], pode ser observado nas microfotografias obtidas em MEV (Fig. 4). Nessas mostra-se também a presença de microtrincas que não foram observadas a olho nu nos corpos cerâmicos.

Os ensaios tecnológicos apresentaram absorção de água (Tabela V), entre 13,23\% para 9.R1 e 30,4\% para 9.R5+5F. De acordo com as especificações para tijolos cerâmicos esses valores devem estar entre 8 e $25 \%$ [47]. Assim, a maior parte dos corpos cerâmicos aqui obtidos cumpre essas especificações. Entretanto, observações macroscópicas dos corpos de prova mostraram a presença de microfissuras que podem ser relacionadas aos corpos de prova com maiores valores de absorção de água, como o caso da amostra 9.R5+5F. Entretanto, as proporções de absorção de água podem ser relacionadas com a presença das microfissuras e com a porosidade dos corpos cerâmicos.

A retração linear (Tabela V) mostrou o valor mínimo de $0,28 \%$ para $9 . \mathrm{ACM}+15 \mathrm{~F}$ e máximo de $7,53 \%$ para 1.AM+10F. Entretanto após queima dos corpos R1 houve uma pequena expansão das dimensões em valores próximos a $0,5 \%$.

A resistência mecânica à compressão (Tabela $\mathrm{V}$ ) foi alta sendo, com um mínimo valor de 16,5 MPa para 1.FS+10F e o máximo de 67,2 Mpa para 1.DV. Esses valores são superiores às especificações técnicas para tijolos cerâmicos que indicam valores mínimos de $1 \mathrm{MPa}$ [47].

\section{CONCLUSÕES}

As diferentes técnicas de análises usadas para a caracterização mineralógica, física e química das argilas de três localidades do Quadrilátero Ferrífero, assim como o estudo tecnológico dos corpos cerâmicos obtidos a partir dessas argilas naturais e de misturas com filito, permitiram concluir que as amostras DV do depósito Padre Domingos e R1 do talude ao lado da rodovia, no Sinclinal Moeda usadas no seu estado natural, são as que apresentaram as melhores características tecnológicas e atingiram as especificações técnicas, resultando em corpos cerâmicos sem fraturas e resistências à compressão superiores às normas. Tal fato pode ser atribuído aos maiores teores de $\mathrm{Fe}_{2} \mathrm{O}_{3}$ em DV e, deste óxido em associação com $\mathrm{SiO}_{2}$ em R1. As demais argilas naturais não atingiram as especificações para tijolos cerâmicos, sendo, portanto necessário o uso do aditivo filito muscovítico para obtenção de características tecnológicas adequadas. A adição deste componente com as misturas de $\mathrm{ACM}, \mathrm{DB}, \mathrm{R} 5$ e FS, segundo as proporções de: $\mathrm{ACM}+15 \mathrm{~F}$, $\mathrm{DB}+5 \mathrm{~F}, \mathrm{R} 5+5 \mathrm{~F}, \mathrm{FS}+5 \mathrm{~F}, \mathrm{AM}+5 \mathrm{~F}$, apesar de não ter provocado a fusão total da mistura a $900{ }^{\circ} \mathrm{C}$ resultou em corpos com características físicas e mecânicas adequadas à indústria de cerâmica vermelha. Esses corpos cerâmicos apresentaram menor retração linear, maior resistência à compressão e ausência de fissuras.

\section{AGRADECIMENTOS}

Os autores agradecem a contribuição financeira da FAPEMIG e do CNPq e a colaboração técnica dos laboratórios de: materiais cerâmicos, difração de raios X, fluorescência de raios $\mathrm{X}$, microscopia eletrônica de varredura e geoquímica ambiental, do Departamento de Geologia; BET e de tratamento de minérios do Departamento de Minas; de análises térmicas do Departamento de Química e de ensaios mecânicos do Departamento de Metalurgia da Universidade Federal de Ouro Preto (UFOP). Agradecemos ainda o suporte técnico do Laboratório de Ensaios Cerâmicos do Departamento de Petrologia e Metalogenia, Instituto de Geociências e Ciências Exatas, Universidade Estadual Paulista.

\section{REFERÊNCIAS}

[1] H. Gorceix, "Bacias terciárias de água doce nos arredores de Ouro Preto (Gandarela e Fonseca), Minas Gerais, Brasil" Anais da Escola de Minas de Ouro Preto, Ouro Preto 3 (1884) 95 .

[2] O. A. Derby, "The Serra do Espinhaço", J. Geology 14, 3 (1906) 374.

[3] O. Barbosa "Resumo da geologia de Minas Gerais", B.DSGG, Belo Horizonte 3 (1934) 40.

[4] E. P. Scorza "Ocorrência de cinábrio em Dom Bosco, Minas Gerais", Notas preliminares e estudos, DGM-DNPM, Rio de Janeiro, RJ 18 (1939) 1.

[5] P. W. Guild "Geology and mineral resources of the Congonhas do Campo District, Minas Gerais, Brazil", Washington, U.S. Geological Survey Professional Paper 690 (1957) 90. 
[6] R. F. Johnson, "Geology and ore deposits of the Cachoeira do Campo, Dom Bosco and Ouro Branco Quadrangles, Minas Gerais, Brazil", Washington, U. S. Geological Survey Professional Paper 341-B (1962) 37.

[7] J. B. Pomerene "Geology and ore deposits of Belo Horizonte, Itabira and Macacos Quadrangles, Minas Gerais, Brazil", Washington, U.S. Geological Survey Professional Paper 341-D (1964) 84.

[8] J. V. N. Dorr, "Physiografic, stratigraphic and structural development of the Quadrilátero Ferrífero, Minas Gerais, Brazil”, Washington, US Geological Survey Professional Paper 641C (1969) 58.

[9] C. V. D’Elboux, C. M. Ferreira, “Topázio na região de Ouro Preto", Boletim Dep. Geologia, UFOP, Publ. Especial 1 (1975) 73.

[10] C. M. Ferreira, "Vulcanismo ácido no Quadrilátero Ferrífero e sua relação com algumas ocorrências minerais", in SBG - Núcleo de Minas Gerais 3, Anais Simp. Geologia de Minas Gerais, Belo Horizonte, MG 3 (1983) 128.

[11] A. F. D. C. Varajão, "Gênese das Jazidas de caulinita e bauxita de Vargem dos Óculos, Quadrilátero Ferrífero, MG", Diss. Mestrado, Instituto de Geociências, USP, S. Paulo, SP (1988) 168.

[12] A. F. D. C. Varajão, B. Boulange, A. J. Melfi, "The Petrologic evolution of the facies in the kaolinite and bauxite deposits of Vargem dos Óculos, Quadrilátero Ferrífero, Minas Gerais, Brazil”, Travaux 19, 22 (1989) 137.

[13] A. F. D. C. Varajão, B. Boulange, A. J. Melfi, "Caracterização das unidades morfológicas e formações superficiais nas adjacências das jazidas de caulinita e bauxita de Vargem dos Óculos, Quadrilátero ferrífero, MG”, Rev. Esc. Minas 42, 1 (1989b) 14.

[14] A. F. D. C. Varajão, B. Boulangé, A. J. Melfi “Evolução morfológica, mineralógica e química da jazida de bauxita de Vargem dos Óculos, Quadrilátero Ferrífero, MG” Anais Cong. Bras. Geoquímica, Rio de Janeiro, RJ 2 (1989c) 393.

[15] A. F. D. C. Varajão, B. Boulangé, A. J. Melfi, "Caracterização morfológica, mineralógica e química das fácies estruturais da jazida de caulinita de Vargem dos Óculos, Quadrilátero Ferrífero, MG” Rev. Bras. Geociências 20, 1-4 (1990) 75.

[16] A. D. Varajão, L. A. Rocha, B. Boulangé, A. P. A. M. Moreira "Colluvial features of the clay deposits of Moeda Syncline, Quadrilátero Ferrífero, Minas Gerais, Brazil", Zentralblatt Geol. Palaont. 7/8 (2000) 957.

[17] A. F. D. C. Varajão, R. J. Gilkes, R. D. Hart, "The relationships between kaolinite crystal properties and the origin of materials for a Brazilian kaolin deposit", Clays Clay Minerals 49 (2001) 44.

[18] M do C. Santos, "Caracterização dos depósitos argilosos da porção centro-sul do Sinclinal Moeda, Quadrilátero Ferrífero, MG: macromorfologia, micromorfologia, cristaloquímica, gênese e considerações industriais", Tese Dr., Dep. Geologia, Escola de Minas, UFOP, Ouro Preto, MG (2003) 199.

[19] M. D. C. Santos, A. F. D. C. Varajão, J. Yvon, "Genesis of clayey bodies in Quadrilatero Ferrífero, Minas Gerais,
Brazil", Catena 55 (2004) 277.

[20] M. D. C. Santos, A. F. D. C. Varajão, "Sedimentation and Pedogenic features in a clay deposit in Quadrilátero Ferrífero, Minas Gerais, Brazil", Anais Acad. Bras. Ci. 76 (2004) 147.

[21] M. do C. Santos, "Gênese dos corpos argilosos do Morro do Caxambu e da Mina do Vermelhão, Sinclinal Dom Bosco, Quadrilátero Ferrífero, Minas Gerais”, Diss. Mestrado, Dep. Geologia, UFOP, Ouro Preto, MG (1998) 176.

[22] M. D. C. Santos, A. F. D. Varajão, P. T. A. Castro, A. P. A. Moreira "Gênese dos "depósitos argilosos" da Mina do Vermelhão e do Morro do Caxambu, Sinclinal Dom Bosco, M.G", Anais Simp. Geol. Centro-Oeste, Simp. Geol. Minas Gerais, SBG-Núcleo Brasília, Res. Exp. (1999) 40.

[23] A. F. D. C. Varajão, M. D. C. Santos "Facies distribution and pedogenic evolution of the clayey deposit of Caxambu Hill, Minas Gerais, Brazil" Anais $12^{\text {th }}$ Int. Clay Conf., Bahía Blanca, A Clay Odyssey, Elsevier Sci. B. V. (2001) 171-178 [24] A. B. Luz, F. A. F. Lins, J. M. Coelho, "Feldspato", in A. B. Luz, F. A. F. Lins (Eds.), Rochas e minerais industriais: usos e especificações, $2^{\text {a }}$ Ed., Rio de Janeiro, RJ CETEM/ MCT (2008) 990.

[25] A. P. F. Gorini, A. R. Correa "Cerâmica para revestimentos", BNDES, Rio de Janeiro, RJ, 10 (1999) 201-252.

[26] J. F. M. Motta, M. C. Junior, L. C. Tanno "Panorama das matérias-primas utilizadas na indústria de revestimentos cerâmicos: desafios ao setor produtivo", Ceram. Ind. 3, 4-6 (1998) 30-38.

[27] G. M. Bustamante, J. C. Bressiani, "A indústria cerâmica brasileira", Ceram. Ind. 5, 3 (2000) 31-36.

[28] ABNT - Associação Brasileira de Normas Técnicas, NBR 6459, "Solo - Determinação do limite de liquidez", Rio de Janeiro, RJ (1984) 1.

[29] ABNT - Associação Brasileira de Normas Técnicas, NBR 7180, "Solo - Determinação do limite de plasticidade", Rio de Janeiro, RJ (1984) 1.

[30] L. A. Gaspar Jr., "Adição experimental de novos materiais às argilas da região do pólo cerâmico de Santa Gertrudes (SP)", Tese Dr., Dep. Petrologia e Metalogenia, Instituto de Geociências e Ciências Exatas, UNESP, Rio Claro, SP (2003) 170.

[31] SEBRAE - Serviço Brasileiro de Apoio às Micro e Pequenas Empresas, "Cerâmica vermelha para construção: telhas, tijolos e tubos", SEBRAE/SPEM, Rel. (2008) 93.

[32] ABNT - Associação Brasileira se Normas Técnicas, NBR 8947, "Telha cerâmica - Determinação da massa e da absorção de água”, Rio de Janeiro, RJ (1985) 1.

[33] ABNT - Associação Brasileira de Normas Técnicas, NBR 6460, "Tijolo maciço cerâmico para alvenaria Verificação da resistência à compressão - Método de ensaio", Rio de Janeiro, RJ (1984) 1.

[34] R. C. L. Araujo, E. H. V. Rodrigues, E. G. A. Freitas, Cap. 7, "Produtos cerâmicos. Materiais de construção", 1 EDUR, Rio de Janeiro, RJ (2000) 108.

[35] S. Ramos, J. Alexandre, M. G. Alves, V. Vogel, M. Gantos, "A indústria cerâmica vermelha de Campos dos 
Goitacazes e a inclusão social das artesãs da baixada campista através do projeto Caminhos de Barro", Cerâmica 54 (2008) 280.

[36] E. L. Bittencourt, J. C. B. Júnior, M. D. M. Silvestre, "Metodologia experimental para desenvolvimento de uma formulação de revestimento poroso (Classificação BIII) em processo de monoporosa através da programação linear", Ceram. Ind. 6, 4 (2001) 35.

[37] F. Watari, J. Van Landuyt, P. Delavignette, S. Amelinckx, N. Igata, "X-Ray peak broadening as a result of twin formation in some oxides derived by dehydration", Physica Status Solidi 73 (1982) 215.

[38] M. D. Moreira "Aplicações dos minerais e rochas industriais", SBG - Soc. Bras. Geologia, Salvador, BA (1994) 87.

[39] A. I. Avgustinik, "Ceramica”, Ed. Reverté, Barcelona, Espanha (1983) 726.

[40] R. C. Mackenzie, "The differential thermal investigation of clays", Mineralogical Soc., London, UK (1957) 456.

[41] Munsell Soil Color Company, Munsell Soil Color
Charts, U.S. Dept. Agriculture, Baltimore, EUA (1975).

[42] H. Naomo, R. Fujiwara, "Micropore formation due to thermal decomposition of acicular microcrystals of a-FeOOH”, J. Colloid Interface Sci. 73, 2 (1980) 406.

[43] J. Morales, J. L. Tirado, M. Macias, "Changes in crystallite size and microstrains of hematite derived from the thermal decomposition of synthetic akaganeite", J. Solid State Chem. 53 (1984) 303.

[44] G. M. da Costa, M. F. de Jesus Filho, "X-ray differential line broadening on tabular hematites", J. Mater. Sci. 27 (1992) 6116.

[45] G. P. Santana, J. D. Fabris, A. T. Goulart, D. P. Santana, "Magnetite and its transformation to hematite in a soil derived from steatite" Rev. Bras. Ci. Solo 25, 1 (2001) 22.

[46] L. Van Vlack, "Propriedades dos materiais cerâmicos", Ed. Edgar Blücher Ltda., S. Paulo, SP (1973) 94.

[47] INMETRO - Instituto Nacional de Metrologia, "Normalização e qualidade industrial, bloco cerâmico (tijolo)", Ministério de Desenvolvimento, Indústria e Comercio Exterior do Brasil (2001) 1.

(Rec. 30/07/2010, Rev. 16/09/2010, 07/11/2010, Ac 18/11/2010) 